\title{
Electron and Ion Acceleration in the Unstable Auroral lonosphere
}

\author{
C. C. Chaston ${ }^{1, *}$, J. W. Bonnell ${ }^{1}$, C. W. Carlson ${ }^{1}$, R. E. Ergun ${ }^{2}$, J. P. McFadden ${ }^{1}$, R. J. Strangeway ${ }^{3}$ and E. J. Lund $^{4}$ \\ ${ }^{1}$ Space Sciences Laboratory, University of California, Berkeley, 97470 CA, USA \\ ${ }^{2}$ Laboratory for Atmospheric and Space Physics, University of Colorado, Boulder, 80303 CO, USA \\ ${ }^{3}$ Institute for Geophysics and Planetary Physics, University of California, Los Angeles, 90095 CA, USA \\ ${ }^{4}$ Space Sciences Center, University of New Hampshire, Durham, 03824 NH, USA
}

Received 8 September 2003

PACS Ref: $94.30 . \mathrm{Tz}$

\begin{abstract}
It is demonstrated that energetic electrons accelerated in the field-aligned electric wavefield of kinetic Alfvén waves in near Earth space destabilize the ionosphere to secondary Alfvén waves via the well known ionospheric feedback instability. From spacecraft observations and simulations it is shown how this instability may drive waves at wavelengths perpendicular to the geomagnetic field of the order of the electron inertial length $\left(\lambda_{\mathrm{e}}\right)$ and ion gyro-radius $\left(\rho_{\mathrm{i}}\right)$. These secondary waves then lead to further electron acceleration and ion trapping. The trapping process results in coherent acceleration up to energies where the ion gyro-radius is equal to half the wavelength of the wave transverse to the geomagnetic field $\left(\lambda_{\perp}\right)$. For sufficiently large wave amplitudes at these scales the orbit of the ions in the wavefield is disrupted and may become stochastic as has been suggested previously from observations by others.
\end{abstract}

\section{Introduction}

Observations of plasmas above the auroral ionosphere invariably contain field-aligned accelerated electrons and transversely accelerated ions [1]. The explanation of these observations has been the focus of auroral plasma physics since spacecraft first flew above aurora. It has been shown that the most intense accelerated ion and electron fluxes are observed simultaneously with oscillating perpendicular electric and magnetic fields which roughly obey $E_{\perp 1} / B_{\perp 2} \gtrsim V_{\mathrm{A}}$ where $V_{\mathrm{A}}$ is the Alfvén speed [2-5]. These oscillations are of course Alfvén waves.

The sources for Alfvén waves above the auroral oval are ultimately magnetospheric. However, the ionosphere in the presence of a convection electric field may become unstable to Alfvén waves due the resonant cavity formed between the ionosphere and the Alfvén speed peak at $\sim 1 \mathrm{Re}$ altitude. This cavity is known as the ionospheric Alfvén resonator (IAR) [6,7] and has been identified from observations $[8,9]$. If the ionospheric conductivity in the ionosphere is non-uniform, an Alfvén wave bouncing within the cavity will reflect from a different ionospheric conductivity with each bounce. For a convecting and oscillatory conductivity in the ionosphere the phasing between the wave and the conductivity may be such that the amplitude of the reflected wave exceeds that of the incident wave. This is the ionospheric feedback instability of the IAR [10-12].

In this report we show how electrons accelerated in a single Alfvénic pulse from the magnetosphere may stimulate the feedback instability. The instability then causes wave growth for Alfvén waves on scales sufficiently

\footnotetext{
*e-mail: ccc@ssl.berkeley.edu
}

small that they strongly interact with ionospheric ions. These ions due the vertical gradient in the geomagnetic field and their enhanced magnetic moment are rapidly accelerated upwards from the ionosphere into near Earth space.

\section{Kinetic Alfvén Wave Model for the IAR}

Observations suggest that waves with scales of the order of the electron skin depth $\left(\lambda_{\mathrm{e}}=c / \omega_{\text {pe }}\right.$ with $\omega_{\text {pe }}$ being the electron plasma frequency) above the auroral oval are not planar but are elliptical and become more circular with decreasing scale [13]. In this region $\lambda_{\mathrm{e}}>\rho_{\mathrm{i}}$ so to study waves on scales where $\lambda_{\perp} \sim \rho_{\mathrm{i}}$ we generalize the plane wave case and define the transverse wavefields as,

$$
\begin{aligned}
& E_{\perp 1}(x, y, z, y)=-k_{\perp 1} \phi(z, t) \cos \left(k_{\perp 1} x\right) \cos \left(k_{\perp 2} y\right), \\
& E_{\perp 2}(x, y, z, y)=k_{\perp 2} \phi(z, t) \sin \left(k_{\perp 1} x\right) \sin \left(k_{\perp 2} y\right), \\
& B_{\perp 1}(x, y, z, y)=k_{\perp 2} \phi(z, t) \sin \left(k_{\perp 1} x\right) \sin \left(k_{\perp 2} y\right), \\
& B_{\perp 2}(x, y, z, y)=k_{\perp 1} \phi(z, t) \cos \left(k_{\perp 1} x\right) \cos \left(k_{\perp 2} y\right)
\end{aligned}
$$

where $k_{\perp 1}=2 \pi / \lambda_{\perp 1}$ and $k_{\perp 2}=2 \pi / \lambda_{\perp 1}$ are orthogonal and independent wavenumbers transverse to the geomagnetic field. Here $A_{\|}(z, t)$ and $\phi(z, t)$ are the wave vector and scalar potentials and $x, y, z$ are coordinates in the two transverse and field-aligned direction respectively with $t$ being time. A snapshot of the wave surface described by Eq. (1) appears like the dimpled surface of a golf ball. These waves become planar when $k_{\perp 1} \geq 0$ or $k_{\perp 2} \geq 0$. Conveniently we find $\nabla_{\perp}=\left(\mathrm{i} k_{\perp 1}, \mathrm{i} k_{\perp 2}\right)$ and $\nabla_{\perp}^{2}=-\left(k_{\perp 1}^{2}+k_{\perp 2}^{2}\right)$ so the model formulation remains similar to that for kinetic plane waves as described by [14] where

$$
\begin{gathered}
\frac{V_{A}^{2}}{1+V_{A}^{2} / c^{2}}\left[1+k_{\perp}^{2} \rho_{i}^{2}\right] \frac{\partial A_{\|}}{\partial z}+\frac{\partial \phi}{\partial t}=0, \\
\left(1+k_{\perp}^{2} \lambda_{e}^{2}\right) \frac{\partial A_{\|}}{\partial t}=-\frac{\partial \phi}{\partial z}\left(1+\frac{k_{\perp}^{2} \rho_{s}^{2}}{1+k_{\perp}^{2} \rho_{i}^{2}}\right) .
\end{gathered}
$$

Here $k_{\perp}^{2}=k_{\perp 1}^{2}+k_{\perp 2}^{2}, \rho_{\mathrm{i}}$ is the ion gyro-radius and $\rho_{\mathrm{s}}$ is the ion acoustic gyro-radii given by $v_{\mathrm{s}} / \Omega_{\mathrm{i}}$ with $v_{\mathrm{s}}$ being the ion acoustic speed and $\Omega_{\mathrm{i}}$ the ion cyclotron frequency. Equations (2) and (3) describe the variation in time and position along a geomagnetic fieldline of a dispersive Alfvén wave assumed periodic in the transverse directions as defined by Eq. (1). If we now assume periodicity along 
the geomagnetic field and in time then these waves approximately obey the dispersion relation,

$\omega / k_{\|}=V_{A} \sqrt{\frac{1+k_{\perp}^{2}\left(\rho_{i}^{2}+\rho_{s}^{2}\right)}{1+k_{\perp}^{2} \lambda_{e}^{2}}}$.

The field-aligned wave magnetic field is zero, which from Faraday's law requires $k_{\perp 1} / k_{\perp 2}=E_{\perp 1} / E_{\perp 2}$ and the geomagnetic field-aligned electric field is,

$$
\begin{aligned}
E_{\|} & =-\frac{\partial A_{\|}}{\partial t}-\frac{\partial \phi}{\partial z} \\
& =\frac{\partial A_{\|}}{\partial t}\left[\frac{\left(1+k_{\perp}^{2} \lambda_{e}^{2}\right)\left(1+k_{\perp}^{2} \rho_{i}^{2}\right)-\left(1+k_{\perp}^{2}\left(\rho_{i}^{2}+\rho_{s}^{2}\right)\right)}{\left(1+k_{\perp}^{2}\left(\rho_{i}^{2}+\rho_{s}^{2}\right)\right)}\right] .
\end{aligned}
$$

Consequently, these waves carry a parallel to the geomagnetic field electric field capable of accelerating electrons. The ability of Alfvén waves to cause aurora through the action of the parallel field at scales where $k_{\perp} \rho_{\mathrm{s}}, \lambda_{\mathrm{e}} \sim 1$ has been the topic of numerous studies [15-20].

In the model to be discussed in the remainder of this report, Eqs. (2) and (3) are solved subject to boundary conditions at the magnetospheric end of the simulation domain and the ionosphere. At the magnetospheric end this is given by setting the conductivity equal to the Alfvén conductivity of the fieldline in the local approximation so that $A_{\|} \mu_{\mathrm{o}} \Sigma_{\mathrm{A}}\left(\phi_{\mathrm{o}}(+)-\phi\right)=0$ [18]. This allows the wave to freely pass through the boundary after reflection from the ionosphere. Here $\Sigma_{\mathrm{A}}=1 /\left(\mu_{\mathrm{o}} E_{\perp 1} / B_{\perp 2}\right)$ and the ratio $E_{\perp 1} / B_{\perp 2}$ is given from the Fourier transform of Eqs. (2) and (3) along $\boldsymbol{B}_{\mathrm{o}}$ and Faraday's law as

$$
\begin{aligned}
E_{\perp 1} / B_{\perp 2} & =V_{A} \sqrt{\frac{1+k_{\perp}^{2} \lambda_{e}^{2}}{1+k_{\perp}^{2}\left(\rho_{i}^{2}+\rho_{s}^{2}\right)}}\left(1+k_{\perp}^{2} \rho_{i}^{2}\right) \\
& =E_{\perp 2} / B_{\perp 1} .
\end{aligned}
$$

$\phi_{\mathrm{o}}(t)$ is an applied potential, which allows a wave to be launched down the fieldline from the magnetospheric end. At the ionospheric end we use a boundary condition derived from current continuity $[9,11]$ and given by,

$$
\begin{gathered}
A_{\|}+\mu_{o} \sum_{P} \Phi+\mathrm{i} \mu_{o} \sum_{P 1} \frac{k_{\perp 1} E_{o 1}+k_{\perp 2} E_{o 2}}{k_{\perp}^{2}} \\
-\mu_{o} \sum_{H 1} \frac{k_{\perp 1} E_{o 2}-k_{\perp 2} E_{o 1}}{k_{\perp}^{2}}=0 .
\end{gathered}
$$

Here we have linearized the height integrated Hall and Pedersen conductivities so that $\Sigma_{\mathrm{P}}=\Sigma_{\mathrm{Po}}+\Sigma_{\mathrm{P} 1}$ and $\Sigma_{\mathrm{H}}=\Sigma_{\mathrm{Ho}}+\Sigma_{\mathrm{H} 1}$ and allowed an arbitrary angle between $\mathrm{k}_{\perp 1}$ and the convection electric field, $E_{\mathrm{O}}=\left(E_{\mathrm{O} 1}, E_{\mathrm{O} 2}\right)$, in the ionosphere. This introduces the terms containing both the Hall and Pedersen conductivity in Eq. (7). $\Sigma_{\mathrm{P} 1}$ and $\Sigma_{\mathrm{H} 1}$ are given by the solution of a density continuity equation,

$$
\begin{aligned}
& \left(\frac{\partial}{\partial t}+\mathrm{i}\left(v_{o 1} k_{\perp 1}+v_{o 2} k_{\perp 2}\right)\right) n_{1} \\
& \quad=Q k_{\perp}^{2} A_{\|} / \mu_{o}-R\left(2 n_{o} n_{1}+n_{1}^{2}\right)
\end{aligned}
$$

using the assumption that $\Sigma_{\mathrm{P} 1}=P n_{1}$ and $\Sigma_{\mathrm{H} 1}=H n_{1}$ where $n=n_{\mathrm{o}}+n_{1}$ is ionospheric density. The details of the derivation of this boundary condition can be found in $[10,11]$. Here $v_{\mathrm{o}}$ is the convection velocity given by $\mathbf{E}_{\mathrm{o}} \times \mathbf{B}_{\mathrm{o}}$ with $v_{\mathrm{o} 1}$ and $v_{\mathrm{o} 2}$ components of this velocity along the $k_{\perp 1}$ and $k_{\perp 2}$ directions. $Q=\gamma /(\mathrm{e} \Delta z)$ where $\gamma=1$ is the number of electron-ion pairs produced per electron incident on the ionosphere in the Alfvén wave with $\Delta z=50 \mathrm{~km}$ the thickness of current carrying region of the ionosphere. The electron flux incident on the ionosphere is in this case determined from the wave current $k_{\perp}^{2} A_{\|} / \mu_{\mathrm{o}}$. We use this as an approximation for the electron flux provided by electron acceleration in the wave. It is assumed that the average energy of the electrons accelerated in the wave is $\sim 1 \mathrm{keV}$ which from [21] gives $P=H=10^{-5} \Omega \mathrm{cm}^{-3}$ for $\Sigma_{\mathrm{Po}}=1 \Omega, \quad \mathrm{R}=3.0 \times 10^{-7} \mathrm{~cm}^{3} \mathrm{~s}^{-1}$ is the recombination coefficient describing how rapidly ions and electrons recombine after ionization. The complex terms in the ionospheric boundary condition allows for over-reflection from the ionosphere and wave growth inside the ionospheric Alfvén resonator known as the ionospheric feedback instability $[10,11]$.

The solution of Eqs. (2) and (3) is achieved by the leapfrog technique as described by [18] while Eq. (8) in the ionospheric boundary condition is solved at each time step by a standard 4th order Runga Kutta technique.

\section{Simulation results}

We now use the wave model defined above to simulate wave growth in the IAR due to electron acceleration in the wavefield and the subsequent acceleration of ions. The first step is to define the plasma in which the waves propagate.

The plasma properties employed are appropriate for the polar edge of the auroral oval over the altitude range from 100 to $10,000 \mathrm{~km}$. The model used is shown in Fig. 1 and follows from observations from the FAST satellite [14]. The error bars shown here indicate the range of densities observed at each altitude. The temperature of the oxygen components is modeled in altitude using the same form as that adopted by [22] as $T_{\mathrm{O}+}=1+499 \tanh$ (altitude-100)/ $(2 \mathrm{Re})$ in electron Volts which provides a temperature of $1 \mathrm{eV}$ at $100 \mathrm{~km}$ and $\sim 500 \mathrm{eV}$ at altitudes above $\sim 5 \mathrm{Re}$. For $\mathrm{H}^{+}$ions and electrons the profiles are averaged based on the contribution from ionospheric and plasma sheet densities. The ionospheric profiles are given by $T_{\mathrm{H}+\mathrm{e}-}=1+499$ tanh (altitude-100.) $\times 0.4 /(2 \mathrm{Re})$ and the plasma sheet ion and electron temperatures are taken to be 5 and $1 \mathrm{keV}$, respectively, consistent with observations [14]. This provides the steep temperature gradient above $3000 \mathrm{~km}$ altitude where the contribution of the plasma sheet to the average temperature becomes increasingly important. $\mathrm{He}^{+}$ions are not explicitly included here and their inclusion has little effect on the results to be discussed.

Interferometric observations have revealed that Alfvén waves above the aurora have perpendicular scales from kilometers down to $10 \mathrm{~s}$ of meters [22]. We select perpendicular scales of $\lambda_{\perp \mathrm{i}}=3200,800,200$ and $50 \mathrm{~m}$ in the ionosphere at $100 \mathrm{~km}$ altitude where the ' $i$ ' subscript refers to the ionosphere. From the conservation of 

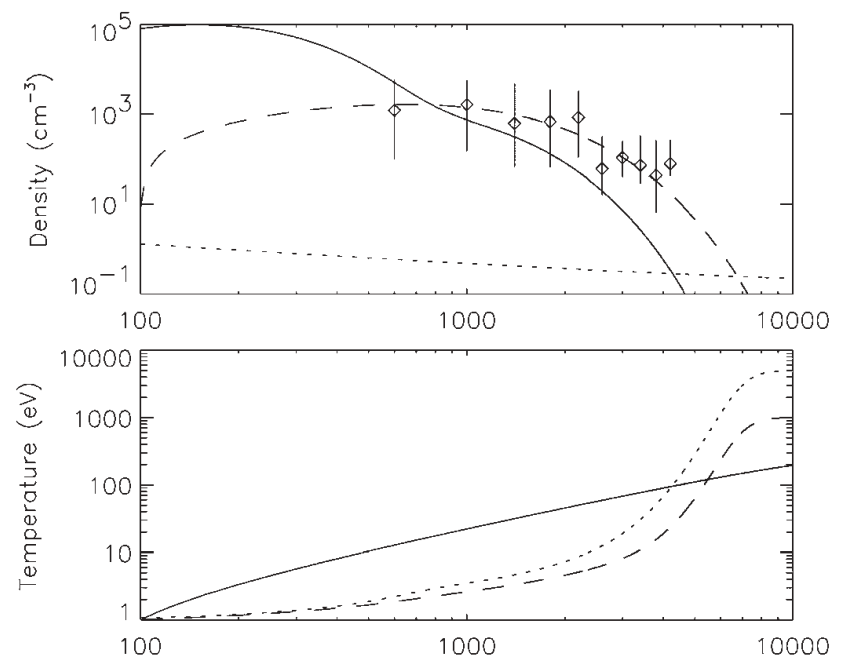

Fig. 1. Altitude dependent model for energetic plasmas above the polar cap boundary. (a) Density of $\mathrm{O}^{+}$ions of ionospheric origin (solid line), $\mathrm{H}^{+}$ ions of ionospheric origin (dashed line) and plasma sheet $\mathrm{H}^{+}$ions (dotted line). The diamonds show median measurements of the total density from FAST observations derived from the electron plasma line in altitude bins of $400 \mathrm{~km}$ width. The error bars show the range of densities in each bin. (b) Temperatures of the same components as described in the text.

magnetic flux we assume that these scales map up the fieldline with the inverse square root of the geomagnetic field strength. For simplicity we have assumed that $k_{\perp 1}$ lies along $\boldsymbol{v}_{\mathrm{O}}$. The magnitude of the convection electric field in the ionosphere is chosen to provide a wave frequency of $k_{\perp 1} v_{\mathrm{o}} \sim 0.6 \mathrm{~Hz}$ in the resonator for each scale. This yields $E_{\mathrm{o}}=100,25,6.25$ and $3.125 \mathrm{mV} / \mathrm{m}$. These fields are within the range of convection fields identified in auroral arcs [23].

The simulation is initiated by a Gaussian pulse in potential at the magnetospheric end of the simulation domain (at an altitude of $10,000 \mathrm{~km}$ ). This disturbance travels towards the ionosphere (at $100 \mathrm{~km}$ ) as an Alfvén wave. When the field-aligned electrons accelerated by the parallel electric field in the wave reach the ionospheric boundary they cause changes in the ionospheric conductivity according to the boundary conditions defined above. Subsequent reflections of this pulse within the ionospheric Alfvén resonator and corresponding ionospheric conductivity variations for the parameters chosen leads to the ionospheric feedback instability [11]. The wave in each case is allowed to grow until it reaches amplitudes of $1 \mathrm{~V} / \mathrm{m}$ at some altitude (usually from 4000 to $8000 \mathrm{~km}$ ). On reaching this condition the instability is turned off by fixing the magnitude of the conductivity oscillations so that wave amplitude remains invariant through the reminder of the simulation. The wave envelopes established in $E_{\perp 1}$ at this time for each scale are shown in Fig. 3(e). Since $\Sigma_{\text {Po }}>\Sigma_{\mathrm{A}}$ the ionosphere is a node, while reflection from the Alfvén speed gradient at the upper boundary of the resonator provides an antinode. These envelopes define the altitude dependency of the wavefields into which test particles are injected. The particles are then traced subject to gravitation and the Lorentz force associated with the wavefield and the geomagnetic dipole.

\subsection{Coherent ion acceleration}

The results from tracing Hydrogen $\left(\mathrm{H}^{+}\right)$(dashed lines) and Oxygen $\left(\mathrm{O}^{+}\right)$ions (solid lines) with initial energies of $1 \mathrm{eV}$

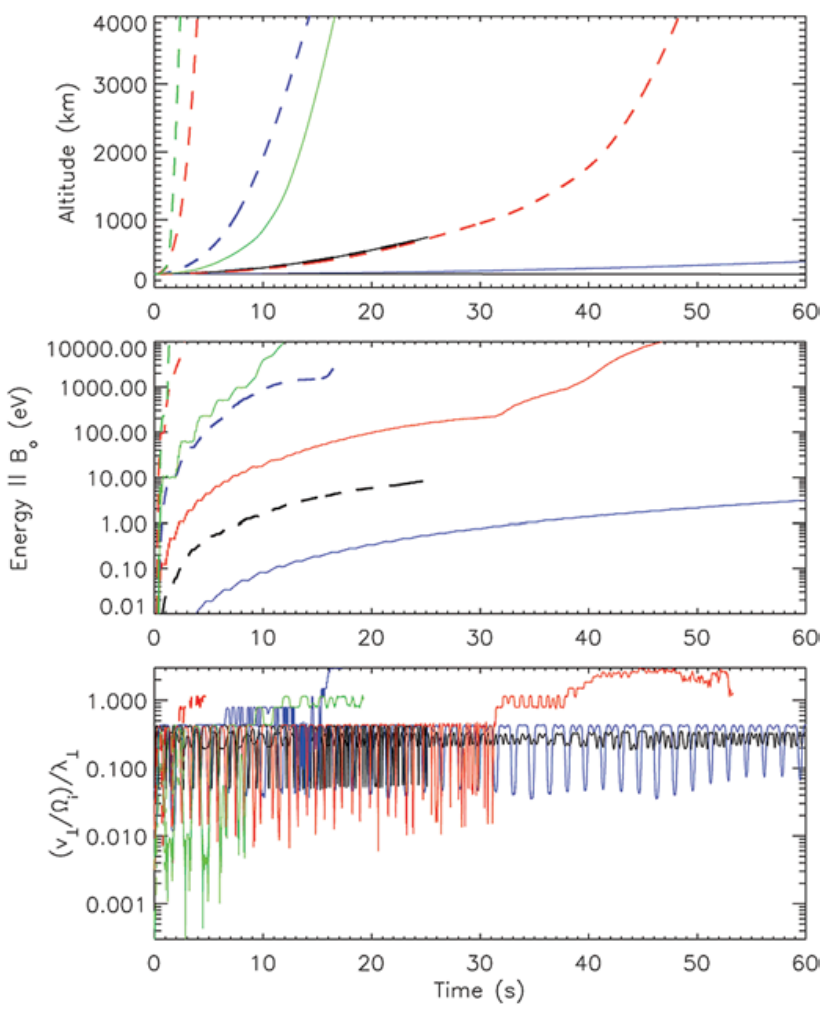

Fig. 2. Wavenumber dependency of ion acceleration. (a) Altitude reached by ions as a function of time. The dashed lines here are for $\mathrm{H}^{+}$ions while the solid lines are for $\mathrm{O}^{+}$. Green lines are for perpendicular wavelengths in the ionosphere of $\lambda_{\perp 1 \mathrm{i}}=\lambda \perp_{2 \mathrm{i}}=3200 \mathrm{~m}$, red is $\lambda_{\perp 1 \mathrm{i}}=\lambda_{\perp 2 \mathrm{i}}=800 \mathrm{~m}$, blue is $\lambda_{\perp 1 \mathrm{i}}=\lambda_{\perp 2 \mathrm{i}}=200 \mathrm{~m}$ and black is $\lambda_{\perp 1 \mathrm{i}}=\lambda_{\perp 2 \mathrm{i}}=50 \mathrm{~m}$. (b) Field-aligned energy of the accelerated ions. (c) Ion gyro-radii normalized by the perpendicular wavelength.

from an altitude of $200 \mathrm{~km}$ are shown in Fig. 2. Part (a) of this figure shows results for each of the wavelengths mentioned above where we have taken $\lambda_{\perp 1}=$ $\lambda_{\perp 2}\left(k_{\perp 1}=k_{\perp 2}\right)$. From Fig. 2(a) it can be seen that the largest scale modeled $\left(\lambda_{\perp \mathrm{i}}=3200 \mathrm{~m}\right.$, green lines) provides the most rapid outflow of ions with $\mathrm{H}^{+}$ions accelerated from $200 \mathrm{~km}$ up to FAST apogee $(4000 \mathrm{~km})$ in 2 or more seconds and $\mathrm{O}^{+}$ions reaching this altitude in 16 or more seconds. Figure 2(b) shows the field-aligned energy of the ions as they stream upwards. The larger $\lambda_{\perp}$ provide larger energies. For the field amplitudes used we find energies extending up to $10 \mathrm{keV}$ over the FAST altitude range. Hydrogen ions gain more energy than oxygen ions. Perpendicular energies while not shown here are generally larger than the field-aligned energy particularly at the lowest altitudes considered where the perpendicular energy can be much larger than the field-aligned energy.

Some understanding of the dependency of the acceleration process on the perpendicular scale of the wave can be gained by considering the ratio of the ion gyro-radii to $\lambda_{\perp}$ as shown in Fig. 2(c). The oscillations in this ratio shown here are in the transverse energy of the ions and at twice the periodicity of the resonator. Here it can be seen that for most of the time range plotted the upper limit of these oscillations occurs when the gyro-radii is roughly half the perpendicular wavelength of the wave. This can be seen more directly by examining the trajectory of the ion over one wave period as shown in Fig. 3(a) and the corresponding energy variations shown in $3(\mathrm{~b})$. The 

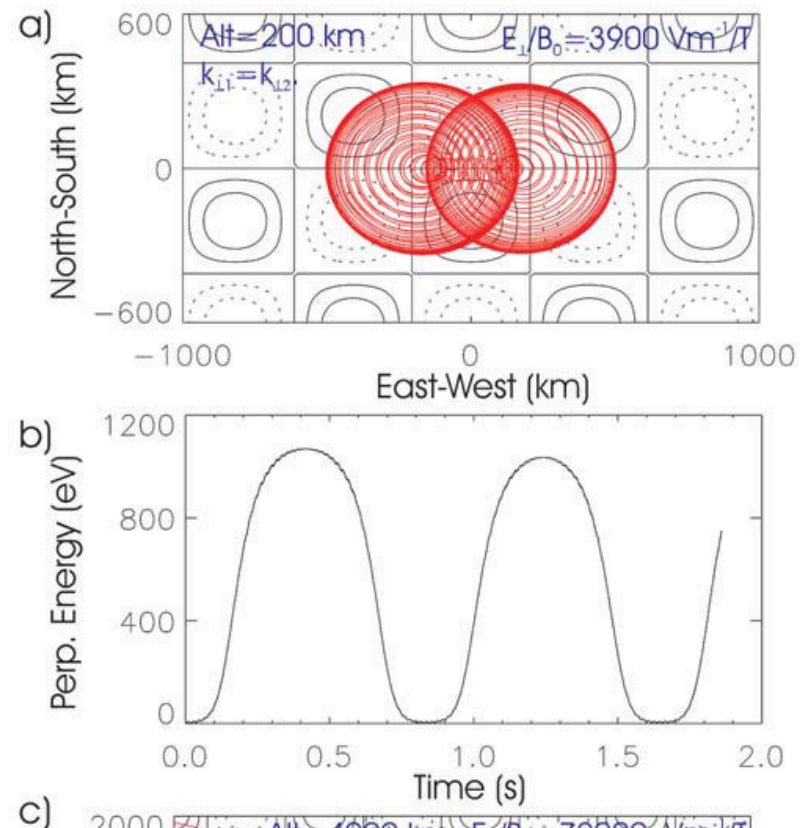

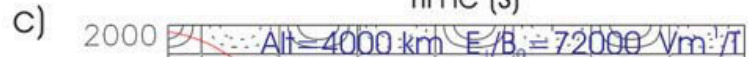
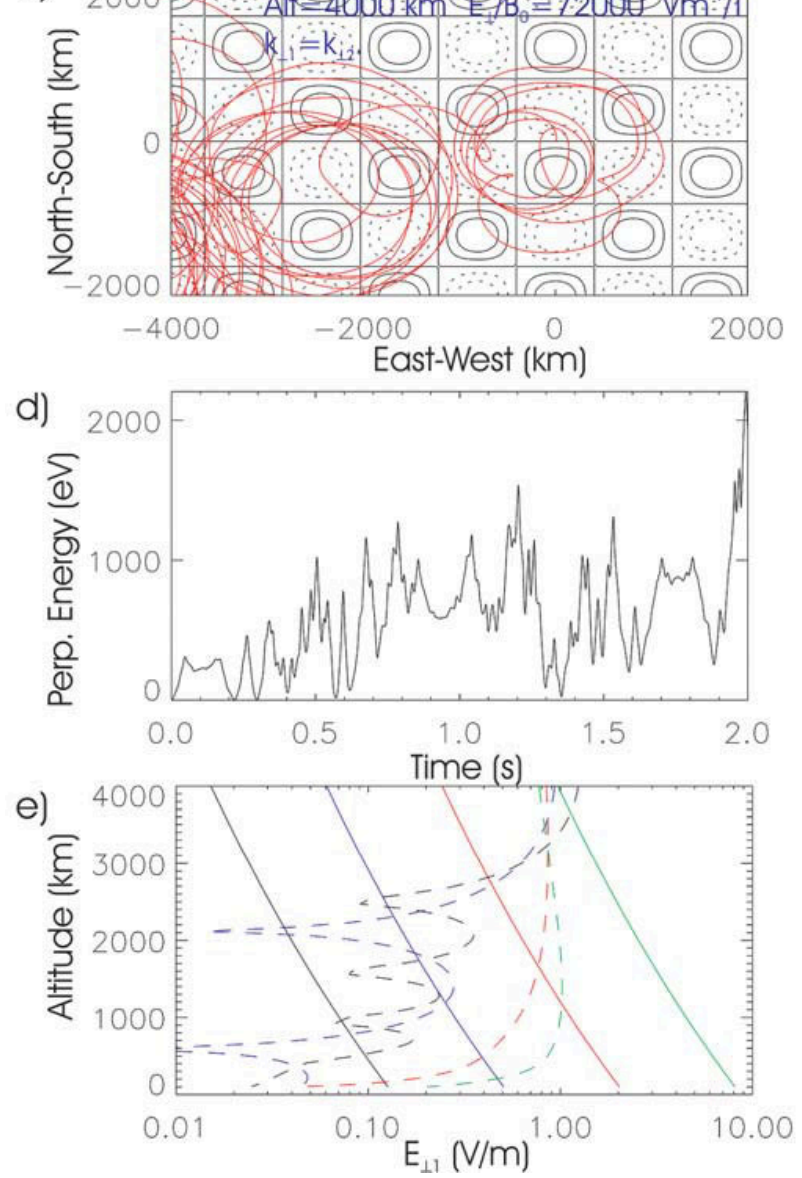

Fig. 3. $\mathrm{O}^{+}$trajectories over slightly more than a wave period for $k_{\perp 1}=k_{\perp 2}$ where $k_{\perp 1 \mathrm{i}}=2 \pi / 800 \mathrm{~m}$. (a) Trajectory of an $\mathrm{O}^{+}$ion at an altitude of $200 \mathrm{~km}$. The contours represent the field-aligned current carried by the waves with dashed contours being upward current and solid contours downwards current. (b) Energy of the $\mathrm{O}^{+}$ion along the trajectory shown in (a). (c) Trajectory of an $\mathrm{O}^{+}$ion at an altitude of $4000 \mathrm{~km}$ with the contours as in part (a). (d) Energy of the $\mathrm{O}^{+}$ion along the trajectory shown in (c). (e) Threshold wave amplitude (solid lines) for stochastic ion motion from Eq. (9) where the dashed lines are the wave amplitude envelopes. Green lines are for perpendicular wavelengths in the ionosphere $\lambda_{\perp 1 \mathrm{i}}=\lambda_{\perp 2 \mathrm{i}}=3200 \mathrm{~m}$, while red is for $\lambda_{\perp 1 \mathrm{i}}=\lambda_{\perp 2 \mathrm{i}}=800 \mathrm{~m}$, blue is for $\lambda_{\perp 1 \mathrm{i}}=\lambda_{\perp 2 \mathrm{i}}=200 \mathrm{~m}$ and black is for $\lambda_{\perp 1 \mathrm{i}}=\lambda_{\perp 2 \mathrm{i}}=50 \mathrm{~m}$. contours shown represent a snapshot of the field-aligned current in the wave. The ion is essentially trapped in the potential of the wave as discussed by [24]. In a uniform background magnetic field and for large wave amplitudes used here this provides an average energy gain over the first wave cycle but not afterwards. Above the auroral oval however, the mirror force associated with the divergent geomagnetic field, if sufficient to overcome gravity, accelerates these oscillating ions upwards. Since $\lambda_{\perp}$ increases with altitude the upward motion of the ions allows them to continue to gain in average energy on each cycle and so contribute to ion outflow [25].

The mass dependency of the acceleration is the result of the finite perpendicular wave scale. For the same gyroradius $\mathrm{H}^{+}$ions have 16 times the energy of $\mathrm{O}^{+}$ions and so in the results shown in Fig. 2, where the gyro-radii limit is reached, the $\mathrm{H}^{+}$ions are more energetic. While this suggests larger scales can provide greater energies, spacecraft observations indicate that the effectiveness of scales larger than $\sim 1 \mathrm{~km}$ for accelerating the ions may be limited. Larger scales require larger wave amplitudes to provide gradients in $E_{\perp}$ over one gyro-radii (particularly in the case of $\mathrm{H}^{+}$) sufficient to yield acceleration up to the gyro-radii limit. Furthermore the ratio of $\lambda_{\perp 1} / \lambda_{\perp 2}$ must be sufficiently close to 1 for any acceleration to occur over reasonable timescales. Given that Freja and FAST observations suggest that these waves become increasing laminar beyond perpendicular scales of the order of $1 \mathrm{~km}$ or $\sim 2 \pi \lambda_{\mathrm{e}}[13,26]$ indicates that the most important scales responsible for ion outflow will be less than $2 \pi \lambda_{\mathrm{e}}$.

\subsection{Stochastic particle motion}

For large enough $E_{\perp}$ and weak enough $\boldsymbol{B}_{\mathrm{o}}$ the particle gyro-motion may be disrupted so that the ions gain energy in excess of that determined by $\lambda_{\perp}$ [27]. To demonstrate this Fig. 3(c) shows the trajectory of an $\mathrm{O}^{+}$ion injected into the $\lambda_{\perp \mathrm{i} 1}=\lambda_{\perp \mathrm{i} 2}=800 \mathrm{~m}$ model wavefield at $4000 \mathrm{~km}$. The proper gyro-motion of the ion is largely absent and it rapidly gains energy as shown in part (d) of this figure whereas in part (a) with a significantly smaller $E_{\perp} / B_{0}$ value the motion remains largely circular.

The theory of stochastic ion acceleration in low frequency waves has been developed by [28]. In the electrostatic case for $k_{\perp} \rho_{\mathrm{i}} \gtrsim 1$ and non-zero $\omega / \Omega_{\mathrm{i}}$, particle motion may become stochastic for a plane wave when the displacement of the guiding center due to polarization drift over one wave period becomes similar to the perpendicular wavelength or when

$E_{\perp} / B_{0} \sim \Omega_{\mathrm{i}} / k_{\perp}$.

This result is independent of the wave mode involved except in the requirement that $B_{0} \gg B_{\perp 1,2}$ which is true above the aurora and is the same condition employed by [27] in explaining ion heating seen in similar waves observed from the Freja spacecraft. Figure 3(e) shows the threshold wave amplitudes required from this relation for stochastic motion for $\mathrm{O}^{+}$ions as a function of altitude for the $\lambda_{\perp}$ considered above. From Eq. (9) the threshold amplitudes for $\mathrm{H}^{+}$are 16 times larger. The dashed lines in this plot show the wave envelopes of the models used. The threshold amplitude for stochastic ion motion is exceeded 
for all scales considered over the altitude range shown for $\mathrm{O}^{+}$ions except at the lowest altitudes. Smaller scales or smaller $\lambda_{\perp}$ for the same wave amplitude are more likely to cause stochastic particle motion. $\mathrm{H}^{+}$ions however will only become stochastic at the highest altitudes considered and for the smaller scales. The presence of higher frequency and multiple scales found in observations [22] however may lower the threshold amplitudes given by Eq. (9) [28].

\section{Conclusions}

1. It has been demonstrated that the feedback instability of the IAR can provide wave growth on scales sufficiently small to transversely accelerate $1 \mathrm{eV} \mathrm{O}^{+}$and $\mathrm{H}^{+}$ions in the ionosphere. These waves can grow for ionospheric convection electric fields as small as a few $\mathrm{mV} / \mathrm{m}$.

2. The ion acceleration process may occur via (a) a coherent process where the ions are trapped in the wave potential and reach energies where there gyro-radii are equal to half the perpendicular wavelength of the wave or via (b) a stochastic process when $E_{\perp} / B_{0}>\Omega_{\mathrm{i}} / k_{\perp}$. It can be expected along an auroral fieldline that the coherent process is most important at ionospheric altitudes particularly for $\mathrm{H}^{+}$ions, while above the ionosphere the stochastic process becomes more important.

3. These acceleration processes may account for observations of ion outflow in Alfvén waves above the aurora [25].

\section{Acknowledgments}

This research was supported by NASA Grant NAG5-12784 and was largely completed at the Chinese University of Hong Kong. Chris Chaston is particularly indebted to Professor Chu Ming Chung and the Department of Physics at the Chinese University.

\section{References}

1. Paschmann, G., Haaland, S. and Truemann, R., "Auroral Plasma Physics," ISSI Space Science Series, ISBN 1-4020-0963-1, March, (2003).

2. Strangeway, R. J. et al., J. Geophys. Res. 105, 129 (2000).

3. Chaston, C. C. et al., J. Geophys. Res. 108, 8003 (2003).

4. Andersson, L. et al., Ann. Geophys. 20, 139 (2002).

5. Knudsen, D. J. et al., J. Geophys. Res. 103, 4171 (1998).

6. Trakhtengertz, V. Y. and Feldstein, A. Y., Geomagn. Aeron. 27, 221 (1987).

7. Lysak, R. L., J. Geophys. Res. 91, 7047 (1986).

8. Grzesiak, M., Geophys. Res., Lett. 27, 923 (2000).

9. Chaston, C. C., J. Geophys. Res. 107, 1351 (2002).

10. Lysak R. L. and Song, Y., J. Geophys. Res. 107, 1029 (2002).

11. Lysak, R. L., J. Geophys. Res. 96, 1553 (1991).

12. Pokhotelov, O. A., J. Geophys. Res. 105, 7737 (2000).

13. Volwerk, J. Geophys. Res. 101, 13335 (1996).

14. Chaston, C. C., Geophys. Res. Lett. 30, 1289 (2003).

15. Hasegawa, A., J. Geophys. Res. 81, 5083 (1976).

16. Goertz, C. K. and Boswell, R. W., J. Geophys. Res. 84, 7239 (1979).

17. Kletzing, C. A., J. Geophys. Res. 99, 11095 (1994).

18. Thompson, B. J. and Lysak, R. L., J. Geophys. Res. 101, 5359 (1996).

19. Hui, C.-H. and Seyler, C. E., J. Geophys. Res. 97, 3953 (1992).

20. Spiro, R. W., J. Geophys. Res. 87, 8215 (1982).

21. Lysak, R. L. and Lotko, W., J. Geophys. Res. 101, 5085 (1996).

22. Stasiewicz, K. Y. et al. Geophys. Res. Lett. 27, 173 (2000).

23. Vondrak, R. R., Geophysical Monograph 25, American Geophysical Union, (Washington DC, 1981), p. 185.

24. Karney, C. F. F. and Bers, A., Phys. Rev. Lett. 39, 550 (1977).

25. Hultqvist, B. et al., "Magnetospheric Ion Sources and Losses," ISSI Space Science Series, ISBN 0-7923-5496-6, September, (1999).

26. Stasiewicz, K. et al., Space Sci. Rev. 92, 423 (2000).

27. Stasiewicz, K. et al., Physica Scripta T84, 60 (2000).

28. Chen, L., Lin, Z. and White, R., Phys. Plasm. 8, 4713 (2001). 